\title{
Expert consensus on perioperative immunotherapy for locally advanced non-small cell lung cancer
}

\author{
Jeong Uk Lim ${ }^{1}$, Hye Seon Kang ${ }^{2}$ \\ ${ }^{1}$ Division of Pulmonary, Allergy and Critical Care Medicine, Department of Internal Medicine, Yeouido St. Mary's Hospital, College of Medicine, \\ The Catholic University of Korea, Seoul, Republic of Korea; ${ }^{2}$ Division of Pulmonary, Allergy and Critical Care Medicine, Department of Internal \\ Medicine, Bucheon St. Mary's Hospital, College of Medicine, The Catholic University of Korea, Seoul, Republic of Korea \\ Correspondence to: Hye Seon Kang, MD, PhD. Division of Pulmonary, Allergy and Critical Care Medicine, Department of Internal Medicine, \\ Bucheon St. Mary's Hospital, College of Medicine, The Catholic University of Korea, 327, Sosa-ro, Wonmi-gu, Bucheon-si, Gyeonggi-do, 14647, \\ Republic of Korea. Email: beyer_kr@catholic.ac.kr. \\ Comment on: Qiu B, Cai K, Chen C, et al. Expert consensus on perioperative immunotherapy for local advanced non-small cell lung cancer. Transl \\ Lung Cancer Res 2021;10:3713-36.
}

Submitted Sep 16, 2021. Accepted for publication Sep 26, 2021.

doi: $10.21037 /$ tlcr-21-764

View this article at: https://dx.doi.org/10.21037/tlcr-21-764

The advent of immune checkpoint inhibitors (ICIs) has rapidly transformed the treatment paradigm for lung cancers. In patients with advanced and locally advanced non-small cell lung cancer (NSCLC) exposed to chemoradiation, the clinical outcomes were improved with ICIs. The use of ICIs for resectable NSCLC, which accounts for $30-40 \%$ among NSCLC, has now expanded into neoadjuvant and adjuvant settings (1). Systemic chemotherapy, including platinum-based regimen, has been the mainstay of perioperative treatment to increase the surgical resection rate, minimize the chances of postoperative recurrence and prolong survival (2). A metaanalysis of 14 trials showed that neoadjuvant chemotherapy resulted in an absolute improvement in recurrencefree survival of $6 \%$ at 5 years (3). However, the survival benefit of adjuvant chemotherapy is $5 \%$ according to the LACE meta-analysis and neoadjuvant chemotherapy when compared with surgery alone $(4,5)$. To improve clinical outcomes, clinical and translational research interventions are underway to utilize ICIs and targeted therapies in the perioperative settings.

A recent expert opinion published in Translational Lung Cancer Research offers a comprehensive review of existing clinical trials of neoadjuvant and adjuvant ICIs with and without chemotherapy, along with management of immunotherapy-related adverse events (irAEs). The experts also commented on the assessment of treatment efficacy.

ICIs in patients with early-stage lung cancer induced greater host immunity and reduced tumor clonal heterogeneity resulting in enhanced antitumor effects (6). Several large-scale studies are ongoing in the neoadjuvant setting as single or dual combinational therapies. Two cycles of nivolumab neoadjuvant ICIs induced a major pathologic response (MPR) of resected tumor (7). However, none of the patients showed an MPR in the PRINCEPS trial, in which only one cycle of atezolizumab was administered (8). Furthermore, the MPR was more frequently observed in the nivolumab plus ipilimumab (50\%) arm than in the nivolumab monotherapy (24\%) arm (9). The combination of neoadjuvant immunotherapy and platinum doublet chemotherapy yielded additional advantages. Phase III trials of various neoadjuvant ICI combination therapies have been proposed, including Checkmate 816, which showed that the median residual population of viable tumor cells in the primary tumor were $10 \%$ vs. $74 \%$ for nivo+chemotherapy $v$. chemotherapy alone (10). In SAKK 16/14 trials, the addition of perioperative durvalumab to neoadjuvant chemotherapy in patients with stage IIIA (N2) NSCLC resulted in an MPR among $62 \%$ of patients and an encouraging 1 -year eventfree survival rate of $73 \%$ (11). Conventional therapies such as chemotherapy might transform cold tumors without an immune response into hot tumors with immunologic activation following exposure to tumor antigens released by dying cancer cells, inducing enhanced therapeutic effects of ICIs (12).

The goal of adjuvant therapy is to treat micrometastatic 
disease and prevent recurrence. Several studies are currently investigating the role of adjuvant immunotherapy in NSCLC. There are ongoing trials of adjuvant therapy with anti PD-1 agents (ANVIL and PEARLS), including two key trials investigating anti-PD-L1 agents in resectable NSCLC (Impower010 and BR31) (13). Results of preplanned interim analysis of Impower010, a randomized phase III trial of adjuvant atezolizumab $v s$. best supportive care (BSC) after adjuvant chemotherapy in patients with early-stage resected NSCLC patients revealed a statistically significant disease-free survival (DFS) following atezolizumab treatment when compared with BSC for patients with PDL1 TC $\geq 1 \%$ stage II-IIIA (14). The ALCHEMIST-nivo trial, which randomized patients with stage IB-IIIA NSCLC without driving mutations to receive 1 year of nivolumab or observation after surgery, has recently completed accrual (15). The results of these studies may have a substantial impact on the clinical management of patients with resectable NSCLC after surgery.

Despite the promising results of perioperative ICIs therapy, several questions have yet to be addressed, including irAEs, which can be serious, and in a few cases, even lifethreatening, resulting in inoperability, delayed surgery, or increased chance of postoperative complications (16). Before starting ICIs, physicians should assess patients' possible risk factors such as autoimmune disease, immunodeficiency disease, and significant infection history. In addition, a regular checkup for possible irAEs using routine blood chemistry tests, pulmonary function tests and ECG during or after neoadjuvant immunotherapy is essential. In a study evaluating the safety of neoadjuvant nivolumab in resectable NSCLC patients, the treatment was not associated with increased perioperative morbidity or mortality. However, half of the video-assisted thoracoscopic surgery/robotic cases were converted to thoracotomy due to hilar lymph node inflammation or fibrosis (17). Further, postoperative irAEs and complications such as lung leakage, bronchopleural fistula, empyema, pulmonary infection and nonspecific pneumonia were reported. The studies to date suggest that neoadjuvant immunotherapy in patients with operable early-stage NSCLC is relatively safe, with incidences of any-grade and $\geq 3$ grade irAEs of $23-57 \%$ and $4.5-13 \%$, respectively. However, these are mostly phase I/II studies with a small sample size and short observation time (16). Large-scale prospective studies with sufficient observation periods are necessary to identify potential risk factors associated with perioperative irAEs. In addition, physicians should be able to manage the possible perioperative irAEs.

Biomarkers predicting postoperative outcomes are essential in order to facilitate neoadjuvant ICI therapies. In the absence of currently available biomarkers that predict response or resistance to ICI therapies, the investigation of other driving mutations using next-generation sequencing (NGS) must be approached with caution. Patients with concurrent EGFR mutations may benefit from receiving EGFR TKIs as perioperative treatment. About $40 \%$ of patients with lung adenocarcinoma in Asia carry EGFR geneactivating mutations. Treatment with neoadjuvant EGFRTKIs resulted in objective response in approximately half of EGFR-sensitive NSCLC patients with tolerable adverse effects (18). Furthermore, the phase III NeoADAURA (NCT04351555) trial of efficacy and safety of osimertinib (with/without chemotherapy) versus chemotherapy plus placebo as neoadjuvant treatment in resectable stage II-IIIB EGFRm NSCLC is still under way (19).

Many of the neoadjuvant chemoimmunotherapy trials evaluated the prognostic value of programmed cell death ligand 1 (PD-L1) or tumor mutation burden (TMB) in clinical outcomes involving locally advanced and metastatic NSCLC. However, in the neoadjuvant setting, PDL1 expression and TMB have shown contrasting results until now (20). Other factors, such as tumor infiltrating lymphocytes (TILs) and immune-related genes (CD 274, PDCD1, CTLA4, B2M, and HLA) were not associated with clinical outcomes including MPR or PFS in neoadjuvant ICIs (21).

To assess treatment response, CT is often used in patients with NSCLC exposed to conventional therapy. The Response Evaluation Criteria in Solid Tumors (RECIST) by CT are valid and consistent in defining response to treatment (22). However, changes in inflammation and interstitial or fibrotic components of tumors affect the CT results, and limit the accuracy of predicting histopathological responses after treatment with neoadjuvant ICIs (23). Some investigators have reported a significant correlation between the pathologic response and the PET response, showing that PET may be a more reliable diagnostic tool for evaluation of treatment response to neoadjuvant ICIs compared with CT (24). In addition, recent studies suggest that analysis of tumor DNA fragments released in the systemic circulation [circulating tumor DNA (ctDNA)] may represent a valuable approach for monitoring tumor burden in NSCLC patients after surgery or predict pathologic tumor regression after neoadjuvant therapies (25). 
In summary, perioperative immunotherapy for resectable NSCLC is promising, but its efficacy and safety have yet to be investigated. Neoadjuvant therapy may delay the timing of surgery, and risk of surgical complications such as hilar fibrosis may not be underestimated. Further, irAEs may occur during and after the treatment, especially when dual ICIs are used. In addition, the assessment of pseudoprogression or hyperprogression before surgery requires in-depth studies and sufficient clinical data. Finally, consensus on patient selection criteria for perioperative ICIs, selection of an appropriate ICI, the number of cycles, and timing of treatment response assessment should be based on vigorous discussion by the experts.

In this "Expert consensus on perioperative immunotherapy for local advanced non-small cell lung cancer", a group of eminent international specialists involved in the treatment of lung cancer present a comprehensive and detailed review of the role of state-of-the art perioperative ICIs in locally advanced resectable NSCLC, discussing the lights and shadows of ICIs, either in AEs during or after ICI treatment and surgical complications, as well as the therapeutic efficacy in clinical trials. We hope that the readers of Translational Lung Cancer Research will find this special series useful in their clinical practice and as an inspiration for further research efforts.

\section{Acknowledgments}

Funding: None.

\section{Footnote}

Provenance and Peer Review: This article was commissioned by the editorial office, Translational Lung Cancer Research. The article did not undergo external peer review.

Conflicts of Interest: Both authors have completed the ICMJE uniform disclosure form (available at https://dx.doi. org/10.21037/tlcr-21-764). The authors have no conflicts of interest to declare.

Ethics Statement: The authors are accountable for all aspects of the work in ensuring that questions related to the accuracy or integrity of any part of the work are appropriately investigated and resolved.

Open Access Statement: This is an Open Access article distributed in accordance with the Creative Commons
Attribution-NonCommercial-NoDerivs 4.0 International License (CC BY-NC-ND 4.0), which permits the noncommercial replication and distribution of the article with the strict proviso that no changes or edits are made and the original work is properly cited (including links to both the formal publication through the relevant DOI and the license). See: https://creativecommons.org/licenses/by-nc-nd/4.0/.

\section{References}

1. Vansteenkiste J, Wauters E, Reymen B, et al. Current status of immune checkpoint inhibition in early-stage NSCLC. Ann Oncol 2019;30:1244-53.

2. Horita N, Miyazawa N, Morita S, et al. Preoperative chemotherapy is effective for stage III resectable non-small-cell lung cancer: metaanalysis of 16 trials. Clin Lung Cancer 2013;14:488-94.

3. NSCLC Meta-analysis Collaborative Group. Preoperative chemotherapy for non-small-cell lung cancer: a systematic review and meta-analysis of individual participant data. Lancet 2014;383:1561-71.

4. Pirker R. Adjuvant chemotherapy in patients with completely resected non-small cell lung cancer. Transl Lung Cancer Res 2014;3:305-10.

5. Salvà F, Felip E. Neoadjuvant chemotherapy in earlystage non-small cell lung cancer. Transl Lung Cancer Res 2013;2:398-402.

6. McGranahan N, Furness AJ, Rosenthal R, et al. Clonal neoantigens elicit $\mathrm{T}$ cell immunoreactivity and sensitivity to immune checkpoint blockade. Science 2016;351:1463-9.

7. Forde PM, Chaft JE, Smith KN, et al. Neoadjuvant PD-1 Blockade in Resectable Lung Cancer. N Engl J Med 2018;378:1976-86.

8. Besse B, Adam J, Cozic N, et al. 1215O - SC Neoadjuvant atezolizumab (A) for resectable non-small cell lung cancer (NSCLC): Results from the phase II PRINCEPS trial. Ann Oncol 2020;31:S794-5.

9. Cascone T, William WN Jr, Weissferdt A, et al. Neoadjuvant nivolumab or nivolumab plus ipilimumab in operable non-small cell lung cancer: the phase 2 randomized NEOSTAR trial. Nat Med 2021;27:504-14.

10. Spicer J, Wang C, Tanaka F, et al. Surgical outcomes from the phase 3 CheckMate 816 trial: Nivolumab (NIVO) + platinum-doublet chemotherapy (chemo) vs chemo alone as neoadjuvant treatment for patients with resectable non-small cell lung cancer (NSCLC). J Clin Oncol 2021;39:8503.

11. Rothschild SI, Zippelius A, Eboulet EI, et al. SAKK 16/14: 
Durvalumab in Addition to Neoadjuvant Chemotherapy in Patients With Stage IIIA(N2) Non-Small-Cell Lung Cancer-A Multicenter Single-Arm Phase II Trial. J Clin Oncol 2021;39:2872-80.

12. Sharma P, Allison JP. The future of immune checkpoint therapy. Science 2015;348:56-61.

13. Sands JM, Mandrekar SJ, Kozono D, et al. Integration of immunotherapy into adjuvant therapy for resected non-small-cell lung cancer: ALCHEMIST chemo-IO (ACCIO). Immunotherapy 2021;13:727-34.

14. Wakelee HA, Altorki NK, Zhou C, et al. IMpower010: Primary results of a phase III global study of atezolizumab versus best supportive care after adjuvant chemotherapy in resected stage IB-IIIA non-small cell lung cancer (NSCLC). J Clin Oncol 2021;39:8500.

15. Chaft JE, Dahlberg SE, Khullar OV, et al. EA5142 adjuvant nivolumab in resected lung cancers (ANVIL). J Clin Oncol 2018;36:TPS8581.

16. Ni J, Huang M, Zhang L, et al. Clinical recommendations for perioperative immunotherapy-induced adverse events in patients with non-small cell lung cancer. Thorac Cancer 2021;12:1469-88.

17. Bott MJ, Yang SC, Park BJ, et al. Initial results of pulmonary resection after neoadjuvant nivolumab in patients with resectable non-small cell lung cancer. J Thorac Cardiovasc Surg 2019;158:269-76.

18. Shi X, Dong X, Zhai J, et al. Current Evidence of the Efficacy and Safety of Neoadjuvant EGFR-TKIs for Patients With Non-small Cell Lung Cancer. Front Oncol 2021;11:608608.

Cite this article as: Lim JU, Kang HS. Expert consensus on perioperative immunotherapy for locally advanced non-small cell lung cancer. Transl Lung Cancer Res 2021;10(9):37093712. doi: $10.21037 /$ tlcr-21-764
19. Tsuboi M, Weder W, Escriu C, et al. P03.02 Neoadjuvant Osimertinib with/without Chemotherapy vs Chemotherapy for EGFR Mutated Resectable NSCLC: NeoADAURA. J Thorac Oncol 2021;16:S258.

20. Gutierrez-Sainz L, Cruz-Castellanos P, Higuera O, et al. Neoadjuvant Chemoimmunotherapy in Patients with Resectable Non-small Cell Lung Cancer. Curr Treat Options Oncol 2021;22:91.

21. Shu CA, Gainor JF, Awad MM, et al. Neoadjuvant atezolizumab and chemotherapy in patients with resectable non-small-cell lung cancer: an open-label, multicentre, single-arm, phase 2 trial. Lancet Oncol 2020;21:786-95.

22. Schwartz LH, Litière $S$, de Vries E, et al. RECIST 1.1-Update and clarification: From the RECIST committee. Eur J Cancer 2016;62:132-7.

23. William WN Jr, Pataer A, Kalhor N, et al. Computed tomography RECIST assessment of histopathologic response and prediction of survival in patients with resectable non-small-cell lung cancer after neoadjuvant chemotherapy. J Thorac Oncol 2013;8:222-8.

24. Tao X, Li N, Wu N, et al. The efficiency of 18 F-FDG PET-CT for predicting the major pathologic response to the neoadjuvant PD-1 blockade in resectable nonsmall cell lung cancer. Eur J Nucl Med Mol Imaging 2020;47:1209-19.

25. van der Leest $P$, Hiddinga B, Miedema A, et al. Circulating tumor DNA as a biomarker for monitoring early treatment responses of patients with advanced lung adenocarcinoma receiving immune checkpoint inhibitors. Mol Oncol 2021. [Epub ahead of print]. doi: 10.1002/1878-0261.13090. 